\title{
Images and imagination: the role of figures in plant cell and molecular biology publications
}

\author{
C. Neal Stewart Jr.
}

Received: 12 December 2013/Revised: 15 January 2014/Accepted: 30 January 2014/Published online: 15 February 2014 (C) Springer-Verlag Berlin Heidelberg 2014

One of the current mantras in research publishing is that science papers should "tell a story." I have been known to utter this phrase to my own trainees in the form of a strong suggestion. Current wisdom states that telling a cogent story through a scientific publication is an important vehicle to render research findings understandable-not only to reviewers and primary readers of the papers, i.e., other scientists, but also to science writers and the public. After all, a paper with a novel finding that tells a good story can potentially get published in a journal with a high impact factor and will be cited at high frequency. And if these conventional research metrics are not enough to spur on the storytelling, we are now reminded of "altmetrics" (see http://altmetrics.org/manifesto) such as the number of posts on blogs, Facebook and popular media articles. Of course, one problem about telling a story is that stories, by their nature, are often fictional. Research journal articles fall into the genre of non-fiction. The temptation to embellish or "tell a tall tale," ever-present in the quest of storytelling, cannot be tolerated in science papers.

"A picture is worth a thousand words" goes the old saying. Historically, illustrations, graphs, micrographs and other types of figures in papers were used to show key data that would help the reader to understand the most important findings in a study. The same is true today, but in the digital age, molecular data in gels, blots and photographic images are easily manipulated with a few mouse swipes and keystrokes. In addition, raw data images included in

Communicated by G. Hahne.

C. N. Stewart Jr. ( $($ )

Department of Plant Sciences, University of Tennessee,

Knoxville, TN 37996, USA

e-mail: nealstewart@utk.edu older techniques, such as northern blot analysis has been replaced by real-time RT-PCR summary data. Papers published in Plant Cell Reports are typically awash with digital data and images, which should give us pause for a reality check. Back in the old analog days of Kodachrome and Polaroids, what you saw was truth-the raw data. The common adage, "typical results are shown" was usually interpreted as "best results are shown," but at least the reader could assume that the results were real, and that they were, to some degree, represented by the image. Sadly, programs such as Photoshop render this assumption obsolete. Readers, reviewers and editors should be vigilant to assess if "typical results" are actual results at all.

Fanelli's (2009) meta-analysis of research misconduct surveys found that $1.97 \%$ of scientists admitted to falsification or fabrication. More shocking is that scientists admitted that they had observed that $14.12 \%$ of their colleagues had falsified research (Fanelli 2009). Estimates range as high as $20 \%$ of cell biology papers containing a questionable figure (Pearson 2005). Rossner and Yamada (2004) have succinctly described guidelines about permissible image manipulation. There is no need to recapitulate the details of their discussion here-I encourage all scientists to read their paper. What is important as an overriding research integrity issue is that the images shown on the pages of Plant Cell Reports and other journals should reflect the true nature of the data and the experiments performed. For example, if a gel contained 12 lanes, then the best practice is to show the entire gel and only the gel in the panel- the unit of the study should be made clear to the reader. Therefore, images of separate gels should not be spliced together, and if lanes within a gel are removed in an image, it is best practice to state the image was manipulated. A photographic image of plant cells or tissues should be honestly representative. A key guideline is that 
any manipulation of an image should be performed to the entire image and not to just a selected portion. As a good example, the Journal of Cell Biology's guidelines as reported by Rossner and Yamada (2004) seem reasonable: "No specific feature within an image may be enhanced, obscured, moved, removed, or introduced. The grouping of images from different parts of the same gel, or from different gels, fields, or exposures must be made explicit by the arrangement of the figure (e.g., using dividing lines) and in the text of the figure legend. Adjustments of brightness, contrast, or color balance are acceptable if they are applied to the whole image and as long as they do not obscure or eliminate any information present in the original. Nonlinear adjustments (e.g., changes to gamma settings) must be disclosed in the figure legend."

Another example of misuse of images is "image recycling," which is the reprinting of a published figure albeit now with a different caption. For example, an image of callus with marker gene expression - a very pretty example of "typical results" in published paper "A" taken from species " $\mathrm{X}$ "— now appears in paper " $\mathrm{B}$ " showing results from species "Y." Since the same image of callus cannot represent results from both biological species, this image recycling represents a form of plagiarism and falsification. It is fine to reprint original figures (and captions) with the copyright holder's permission, but there should be no intent to deceive.

Plagiarism, data fabrication and falsification are sanctionable research misconduct offenses, and fraudulent image alteration or image recycling in publications and grant proposals is misconduct. Note there is a difference between misconduct and honest error (Resnick and Stewart 2012). Above are examples of misconduct, whereas misinterpreting aspects on a confocal image, for example, would be honest error. Why would a researcher risk his/her career by committing research misconduct? Telling a story with artistic illustrations has tempted many researchers to create data and images that could lead a reader to judge a paper as "elegant," which could be beneficial to the author's career. There seems to be a trend towards beautifying science. That is not totally bad. But over-beautification of science can lead to falsification and fabrication, i.e., research misconduct as typified by the Woo Suk Hwang debacle (Editorial 2006).
What can we do about the situation? I offer three recommendations toward solutions; two are proactive and one is reactive. First, as scientists we need to "let the data speak the truth" and resist the temptation to over-beautify. Include images that are realistic and truly typical of what a scientist might find should they attempt to replicate the study. Go to the extra effort to do the right experiments and make the data presentable, but do not overdo digital enhancements. Second, reviewers and editors should resist the urge to focus too much attention on the aesthetics of figures-especially gels and photographic images. Authors need no new encouragement to manipulate images to enable the acceptance of their papers for publication. Third, journals, including Plant Cell Reports, should subject images to analysis using software to detect image manipulation so that papers containing fraudulent data are not published. There are many software packages that are useful to manipulate images which can also be used to detect manipulation, although there are more sophisticated techniques too (Stamm and Liu 2010).

To conclude, the most important thing that we can as scientists do is ask ourselves, how much do we want to be deceived in research?" I would reckon that none of us want to be deceived at all. Therefore, we should not deceive anyone with our own papers. It is fine to tell a story, let us make sure the stories we tell in the pages of journals are absolutely true.

\section{References}

Editorial (2006) Beautification and fraud. Nat Cell Biol 8: 101-102 Fanelli D (2009) How many scientists fabricate and falsify research? A systematic review and meta-analysis of survey data. PLoS One 4:e5738

Pearson H (2005) Image manipulation; CSI: cell biology. Nature 434:952-953

Resnick DB, Stewart CN Jr (2012) Misconduct versus honest error and scientific disagreement. Account Res 19:56-63

Rossner M, Yamada KM (2004) What's in a picture? The temptation of image manipulation. J Cell Biol 166:11-15

Stamm MC, Liu KJR (2010) Forensic detection of image manipulation using statistical intrinsic fingerprints. IEEE Trans Inf Forensics Secur 5:492-506 